\title{
Extracts of lung cancer cells reveal antitumour antibodies in sera of patients with lung cancer
}

\author{
A.V. Bazhin*, M.S. Savchenko*, O.N. Shifrina", S.Y. Chikina\#, M.A. Goncharskaia", G. Jaques ${ }^{+}$, \\ A.G. Chuchalin", P.P. Philippov*
}

Extracts of lung cancer cells reveal antitumour antibodies in sera of patients with lung cancer. A.V. Bazhin, M.S. Savchenko, O.N. Shifrina, S. Y. Chikina, M.A. Goncharskaia, G. Jaques, A.G. Chuchalin, P.P. Philippov. C) ERS Journals Ltd 2003.

ABSTRACT: The objective of the present study was to reveal antitumour antibodies in sera of patients with small cell lung cancer (SCLC).

The antibodies in sera of patients with SCLC and other tumours were detected by immunoblotting with whole extracts of SCLC cells as the antigen source. Sera of patients with various pulmonological disorders, irradiated during the liquidation of consequences of the Chernobyl nuclear power plant incident (a high-risk group in lung cancer), were also analysed.

The present authors' found that SCLC sera contain a set (pattern) of antitumour antibodies which are rarely detected in sera of patients with cancers different from SCLC and very rarely, if ever, present in sera of healthy individuals. The sensitivity and the specificity of the pattern are equal to $80 \%$ and $91 \%$, correspondingly. In the highrisk group in lung cancer, the frequencies of the antibodies are somewhat lower than the corresponding values in SCLC sera, but significantly larger than those in healthy sera.

The findings of the present study create a basis for clinical application of the antitumour antibodies described.

Eur Respir J 2003; 21: 342-346.

\begin{abstract}
*Laboratory of Biomedicine, Dept of Cell Signalling, A.N. Belozersky Institute of Physico-Chemical Biology, Moscow State University, " Pulmonology Research Institute and "Laboratory of Immunochemistry, Institute of Carcinogenesis, N.N. Blokhin Cancer Research Centre, Moscow, Russia. ${ }^{+}$Klinik für Hämatologie, Onkologie und Immunologie, Klinikum der PhilippsUniversität, 35033 Marburg, Germany.

Correspondence: A.V. Bazhin, Laboratory of Biomedicine, Dept of Cell Signalling, A.N. Belozersky Institute of Physico-Chemical Biology, Moscow State University, Moscow, Russia. Fax: 70959390978

E-mail: bazhin@belozersky.msu.ru
\end{abstract}

Keywords: Antitumour antibodies, small cell lung cancer, tumour markers

Received: June 52002

Accepted after revision: September 25 2002

This work was supported in part by grants from the Ludwig Institute for Cancer Research and the Russian Foundation for Basic Research $\left(\mathrm{N}^{\circ} 00\right.$ 04-48332).
Serum antibodies against tumour antigens are considered to be a promising tool for early diagnostics of cancer. Thus, cancer-associated neurological syndromes, which are believed to be evoked by antibodies against paraneoplastic antigens expressed in tumours, often appear months or even years before the diagnosis of cancer, sometimes when the tumour is too small to be detected by usual diagnostic techniques [1].

To date, a number of studies demonstrate that antigens present in normal tissues or tumours can be visualised by the sera of patients with various disorders, including cancer. The first examples are the works published in the 1980s that used the sera of patients with retinopathy [2-4] or paraneoplastic dorsal root ganglionopathy [5], associated with small cell lung cancer (SCLC), to detect antigens shared between SCLC and neurones of retina or cerebral cortex, respectively. A much used method of the serological identification of antigens by recombinant expression cloning also employs sera antibodies to identify tumour antigens [6].

In a series of studies, another approach was suggested to detect corresponding antibodies in the sera of patients with cancer. For example, antibodies against paraneoplastic antigens, $\mathrm{Hu}[7,8], \mathrm{Yo}$ and $\mathrm{Ri}$ [9], and recoverin [10], were detected by the corresponding antigens in sera of patients with cancers. In this study, serum antitumour antibodies (ATA) of SCLC patients have been revealed by a whole extract of SCLC cells, instead of the known specific antigens used in these previous studies [7-10]. Using this extract, the present authors' have also screened the sera of patients with cancers different from SCLC and of those with various pulmonological disorders, irradiated during liquidation of consequences of the Chernobyl nuclear power plant incident.

\section{Materials and methods}

\section{Sera}

Sera of patients with various cancers were collected in the clinics of the N.N. Blokhin Cancer Research 
Centre and the Pulmonology Research Institute in Moscow during 1999-2001. Patients with the following cancers were investigated before treatment: SCLC (52 male and eight female), nonsmall cell lung cancer (30 male and seven female), colon cancer (15 male and 10 female), thyroid cancer ( 25 female), breast cancer (30 female), endometrial cancer (25 female), cervical cancer ( 25 female), and ovarian cancer ( 25 female). None of the patients with cancers showed signs of paraneoplastic syndromes according to physical examination and the case histories of the patients. Sera of 36 patients (male) with various pulmonological disorders, irradiated during liquidation of the consequences of the Chernobyl nuclear power plant accident, were collected in the Pulmonology Research Institute, Moscow. Patients of the latter group worked in the Chernobyl area after the nuclear power plant accident for 2-4 months in 1986-1987. None of the patients had any chronic respiratory diseases before the work at Chernobyl. Sera of 115 healthy individuals (male and female) were used as a negative control. Sera were prepared from whole blood and stored at $-70^{\circ} \mathrm{C}$.

\section{Cancer cell lines}

The cells: SCLC cells of National Cancer Institute (NCI)-N417, NCI-H69 and NCI-H82 originally received from American Type Culture Collection; and Sp 20 (murine myeloma), H33 (human hepatoma) and K562 (human leukaemia) cells, a gift from G.I. Abelev's laboratory, Institute of Carcinogenesis, N.N. Blokhin Cancer Research Centre, Moscow, were cultivated in Roswell Park Memorial Institute 1640 medium (Paneko, Moscow, Russia) supplemented with 10\% foetal calf serum at $37^{\circ} \mathrm{C}$ in the atmosphere of $7.5 \%$ carbon dioxide. The cells were collected, washed three times with $20 \mathrm{mM}$ potassium-phosphate buffer $(\mathrm{pH} 7.4)$ and centrifugated $(30,000 \times g, 15 \mathrm{~min})$. The pellet obtained was extracted with $10 \mathrm{mM}$ Tris- $\mathrm{HCl}$ ( $\mathrm{pH} 7.2$ ), containing $1 \mathrm{mM}$ dithiotreitol and proteinase inhibitors. The cells were subcultured twice a week.

\section{Western blotting}

The Western blot analysis of extracts of various cancer cells (see preceding section) as a source of antigens was performed at room temperature after sodium dodecylsulphate polyacrylamide gel electrophoresis in $12 \%$ gel; $0.05 \mathrm{mg}$ of a total protein were applied per track. The gel slabs were electrotransferred to Hybond-C Extra nitrocellulose membranes (Amersham Pharmacia Biotech, Uppsala, Sweden) in Tris-glycine-methanol buffer, $\mathrm{pH}$ 8.3. The membranes were first incubated $(1.5 \mathrm{~h}$, room temperature) with $10 \%(\mathrm{w} / \mathrm{v})$ of delipidated dry milk in $20 \mathrm{mM}$ Tris- $\mathrm{HCl}$ buffer (pH 7.4), containing $500 \mathrm{mM} \mathrm{NaCl}, 0.05 \%$ TWEEN-20 and $1 \mathrm{mM} \mathrm{CaCl}_{2}$ (buffer A), to saturate the sites of nonspecific binding. Then the membranes were incubated at room temperature with sera (the dilution of 1:20 in buffer A) for $12 \mathrm{~h}$, rinsed three times (for $10 \mathrm{~min}$ each) with buffer A, incubated with sheep antihuman immunoglobulin G peroxidase conjugate (Amersham Pharmacia Biotech) at a dilution of 1:500 in buffer $A$ for $1.5 \mathrm{~h}$, rinsed again with $50 \mathrm{mM}$ Tris- $\mathrm{HCl}(\mathrm{pH} 7.6)$ and finally incubated in the same

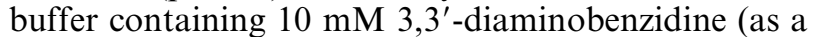
substrate) and $0.01 \%$ hydrogen peroxide.

\section{Statistical analysis}

Comparison between groups was made by Chisquared test. A p-value of $<0.05$ was considered significant. Sensitivity and specificity were expressed using the following equations:

$$
\begin{aligned}
& \text { Sensitivity }=100 \times \text { no. true-positive } / \\
& \text { (no. true-positive }+ \text { no. false-negative) } \\
& \text { Specificity }=100 \times \text { no. true-negative } / \\
& \text { (no. true-negative }+ \text { no. false-positive) }
\end{aligned}
$$

\section{Results}

Figure 1 shows that a whole extract of SCLC cells (line NCI-N417) contains a number of proteins (track E). Some of the proteins cross-react with sera of SCLC patients and visualise 15 typical patterns of bands (fig. 1, tracks 3-17). The extract does not typically cross-react with sera of healthy individuals (for example, track 1), although in some instances, the bands with an apparent molecular weight $\left(\mathrm{M}_{\mathrm{r}}\right)$ above $45 \mathrm{kD}$ can be present (track 2). The cross-reaction is not found in the absence of SCLC sera (not shown). One can also see that anti-p40-p42, the most frequent ATA, are mainly detected in nine different contexts (see fig. 1, tracks 3-11). Anti-p26 and anti-p30 are usually revealed in seven (tracks 5, 7, and 12-16) and five (tracks 4, 7, 9, 14, and 17) different contexts. In addition, anti-p36 (tracks 6, 11, 13, 14 and 15), antip28 (tracks 7, 8, 9, 11 and 16) and anti-p14 (tracks 8, 10,15 and 16) are present in the SCLC sera analysed. In some cases, the extract visualises relatively highmolecular bands with $\mathrm{M}_{\mathrm{r}}>45 \mathrm{kD}$ (for example, see tracks $3,4,7,11,13$ and 15), which can be considered as unspecific since they are also detected occasionally not only in SCLC sera, but also in sera of healthy individuals (track 2). Whole extracts of two other SCLC lines, NCI-H69 and NCI-H82, were also used to visualise ATA in sera of patients with SCLC. When employing these cells, patterns of ATA were similar to those for NCI-N417 cells with the main difference that anti-p36 and anti-p28 were not detected by NCI-H69 extracts at all (data not shown). The frequencies obtained with the SCLC lines used are presented in table 1. In contrast to SCLC sera, sera of healthy individuals contain only anti-p40-p42 and anti-p30 with the corresponding frequencies of $6 \%$ and $3 \%$, respectively (table 1); in both cases, the differences between SCLC sera and that of healthy individuals are statistically significant $(\mathrm{p}<0.0001$ and $\mathrm{p}<0.001$ by $\mathrm{Chi}$-squared test, respectively).

The next step of the work aimed to determine 


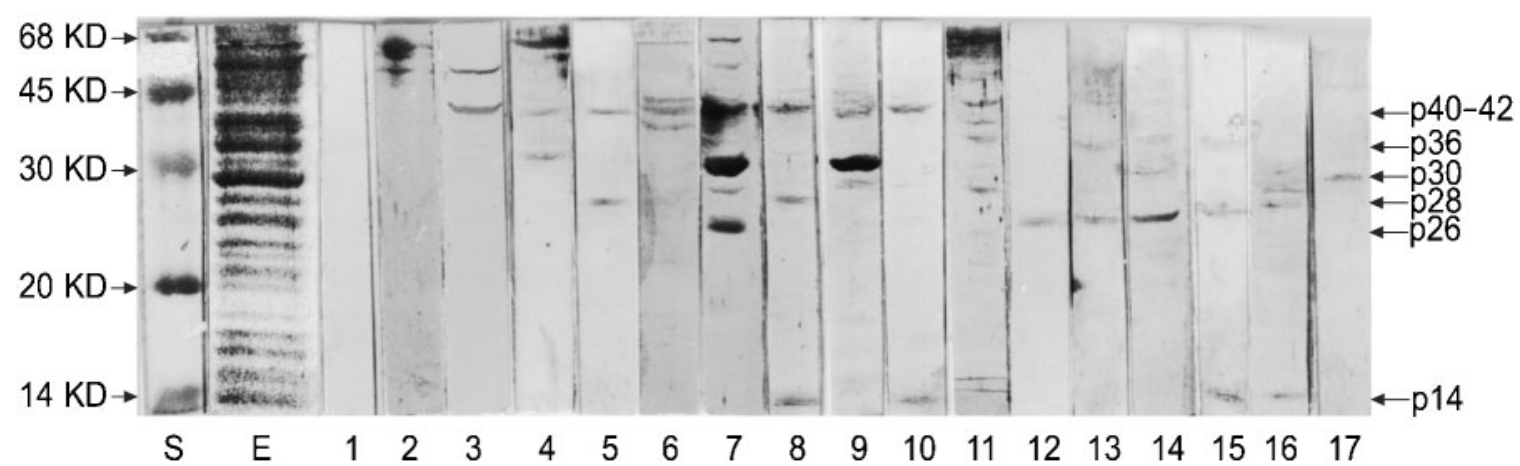

Fig. 1.- Patterns of auto-antibodies visualised in sera of patients with small cell lung cancer (SCLC) by whole extracts of SCLC cells National Cancer Institute (NCI)-N417. Tracks S (molecular weight standard proteins) and E (extracts of NCI-N417 cells) represent blots stained with Panceau S. Tracks 1-17 show blots of extracts of NCI-N417 cells immunostained with sera of healthy individuals (1, 2) or with sera of 15 different patients with SCLC (3-17). Arrows show the positions of the corresponding proteins present in tracks $3-17$.

whether extracts of SCLC cells cross-react with sera of patients with cancers other than SCLC (for this purpose, only one line NCI-N417 was used). From table 2 it can be seen that the frequencies of ATA determined in sera of patients with these cancers are significantly lower than in SCLC sera $(p<0.05)$ with the exception of anti-p30 and anti-p14 for which the differences are nonsignificant ( $\mathrm{p}=0.6$ and 0.07 , respectively).

The serum ATA sensitivity and specificity in SCLC patients compared with healthy individuals are summarised in table 3. All ATA have a specificity higher than $90 \%$. The maximum sensitivity of $59 \%$ is estimated for $\mathrm{p} 40-\mathrm{p} 42$ and it is $<22 \%$ for other ATA. However, the diagnostic value of the combined ATA is significantly better than that of the individual antibodies: the sensitivity rose to $80 \%$ on retention of a high value of the specificity equal to $91 \%$.

It was of interest to know whether whole extracts of

Table 1. - Antitumour antibodies (ATA) revealed in sera of patients with small-cell lung cancer (SCLC) by whole extracts of SCLC cells of lines National Cancer Institute (NCI)-N417, NCl-H69 and NCl-H82

\begin{tabular}{|c|c|c|c|c|c|c|c|c|}
\hline \multirow{2}{*}{$\begin{array}{l}\text { Screening by } \\
\text { extract of }\end{array}$} & \multirow{2}{*}{$\begin{array}{l}\text { Type } \\
\text { of sera }\end{array}$} & \multirow{2}{*}{$\begin{array}{l}\text { Total } \\
\text { cases }^{\#} \mathrm{n}\end{array}$} & \multicolumn{6}{|c|}{ Samples containing ATA against } \\
\hline & & & p40-p42 & p36 & p30 & p28 & p26 & p14 \\
\hline \multirow{2}{*}{ NCI-N417 cells } & SCLC & 60 & $35(59)$ & $8(13)$ & $8(14)$ & $6(10)$ & $13(22)$ & $5(8)$ \\
\hline & Healthy & 115 & $7(6)$ & $0(0)$ & $3(3)$ & $0(0)$ & $0(0)$ & $0(0)$ \\
\hline \multirow[t]{2}{*}{ NCI-H69 cells } & SCLC & 40 & $23(58)$ & $0(0)$ & $3(7.5)$ & $0(0)$ & $10(25)$ & $1(2.5)$ \\
\hline & Healthy & 60 & $0(0)$ & $0(0)$ & $0(0)$ & $0(0)$ & $0(0)$ & $0(0)$ \\
\hline \multirow[t]{2}{*}{ NCI-H82 cells } & SCLC & 40 & $28(70)$ & $1(2.5)$ & $7(18)$ & $4(10)$ & $13(33)$ & $1(2.5)$ \\
\hline & Healthy & 60 & $2(5)$ & $0(0)$ & $0(0)$ & $0(0)$ & $0(0)$ & $0(0)$ \\
\hline
\end{tabular}

Data are presented as n (\%) unless otherwise stated. ${ }^{\#}$ : total amount of individuals investigated are taken as $100 \%$.

Table 2. - Antitumour antibodies (ATA) revealed, by whole extracts of National Cancer Institute (NCI)-N417 cells in sera of patients with cancers other than and with nonmalignant pulmonological disorders

\begin{tabular}{|c|c|c|c|c|c|c|c|}
\hline \multirow[t]{2}{*}{ Type of sera } & \multirow{2}{*}{$\begin{array}{c}\text { Total } \\
\text { cases }^{\#} \mathrm{n}\end{array}$} & \multicolumn{6}{|c|}{ Sera samples containing ATA against } \\
\hline & & $\mathrm{p} 40-\mathrm{p} 42$ & $\mathrm{p} 36$ & $\mathrm{p} 30$ & $\mathrm{p} 28$ & p26 & p14 \\
\hline NSCLC & 37 & $10(28)$ & $3(8)$ & $6(16)$ & $0(0)$ & $3(8)$ & $6(16)$ \\
\hline Breast cancer & 30 & $7(23)$ & $0(0)$ & $0(0)$ & $0(0)$ & $5(17)$ & $0(0)$ \\
\hline Endometrial cancer & 25 & $6(24)$ & $0(0)$ & $5(20)$ & $0(0)$ & $0(0)$ & $0(0)$ \\
\hline Cervical cancer & 25 & $5(20)$ & $0(0)$ & $3(12)$ & $0(0)$ & $1(4)$ & $0(0)$ \\
\hline Ovarian cancer & 25 & $7(28)$ & $0(0)$ & $1(4)$ & $0(0)$ & $2(8)$ & $3(12)$ \\
\hline Thyroid cancer & 25 & $4(16)$ & $0(0)$ & $0(0)$ & $0(0)$ & $0(0)$ & $0(0)$ \\
\hline Colon cancer & 25 & $4(16)$ & $0(0)$ & $3(12)$ & $0(0)$ & $0(0)$ & $0(0)$ \\
\hline Pulmonological disorders & 36 & $13(36)$ & $3(8)$ & $3(8)$ & $0(0)$ & $6(17)$ & $0(0)$ \\
\hline Healthy individuals ${ }^{+}$ & 115 & $7(6)$ & $0(0)$ & $3(3)$ & $0(0)$ & $0(0)$ & $0(0)$ \\
\hline
\end{tabular}

Data are presented as n (\%) unless otherwise stated. ${ }^{\#}$ : total amount of individuals investigated are taken as $100 \%$; ${ }^{\circ}$ : patients were irradiated during liquidation of consequences of the Chernobyl nuclear power plant incident; ${ }^{+}:$the data for healthy individuals, taken from table 1 (screening by NCI-N417 cells,) are presented for comparison. NSCLC: nonsmall cell lung cancer. 
Table 3. - Sensitivity and specificity of antitumour antibodies (ATA) in small cell lung cancer

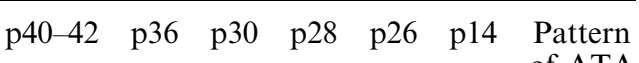

\begin{tabular}{lccccccc}
\hline Sensitivity & 59 & 14 & 14 & 10 & 22 & 8.4 & 80 \\
Specificity & 91 & 100 & 97 & 100 & 100 & 100 & 91 \\
\hline
\end{tabular}

Data are presented as $\%$.

cancer cell lines different from SCLC could cross-react with SCLC sera. To answer this question the cell lines sp 20 (murine myeloma), H33 (human hepatoma) and K562 (human leukaemia) were used. Using the same conditions as were used with extracts of SCLC cells, none of the extracts of these lines visualised any ATA in sera taken from SCLC patients (data not shown).

The data described above show that anti-p40-p42 are most frequent in patients with SCLC and are very rarely present in healthy individuals (table 1). Therefore, it was of particular interest to determine whether ATA could be found in sera of individuals from a high-risk group for lung cancer. For this purpose, extracts of SCLC cells (line NCI-N417) were used to screen sera of 36 patients irradiated during the liquidation of the consequences of the Chernobyl nuclear power plant incident. These patients had various pulmonary disorders such as chronic obstructive or nonobstructive bronchitis and bronchial asthma. According to histological investigation, most of the patients had either mild-to-severe bronchial epithelium dysplasia or focal or diffuse pulmonary fibrosis. As is evident from table 2, the presence of anti-p40-p42 is particularly striking: these ATA occur with a frequency of $36 \%$, six-times higher than that in normal sera. Thus, the anti-p40-p42 frequency holds an intermediate position between sera of SCLC patients $(p=0.04)$ and healthy individuals $(\mathrm{p}<0.0001)$.

\section{Discussion}

SCLC is known to express an array of neuroendocrine cell markers. In particular, several paraneoplastic antigens are described to be associated with SCLC that can cause early development of the corresponding neurological syndromes mediated by antibodies against these antigens [1]. There are data that neurological syndromes may appear months or even years before the cancer becomes evident [4, 11-13]. Although the detection of both the antigens and the corresponding antibodies in sera can be indicative of the presence of SCLC in patients, it should be remembered that, as a rule, no individual marker appears to be of major clinical importance that calls for the use of panels of markers [14].

The current study used whole extracts of SCLC cells, containing a great number of protein antigens, to visualise as far as possible the amount of corresponding antibodies present in sera of patients with SCLC. It turned out that whole extracts of SCLC cells of lines NCI-N417, NCI-H69 and NCI-H82 revealed, in sera of patients with SCLC, antibodies against several proteins with an apparent $M_{r}$ of $40-42,36,30,28$,
26 and $14 \mathrm{kD}$. The most frequently encountered antibodies were those against antigens in the zone $40-42 \mathrm{kD}$, which occurred in SCLC sera with the frequency of $58-70 \%$, whereas these ATA were very rarely, if ever, present in sera of healthy individuals (table 1). The ATA described above were not absolutely specific for SCLC as they could also be detected in sera of patients with cancers other than SCLC. In addition, extracts of other cancer cell lines, such as $\mathrm{sp}$ 20 (murine myeloma), H33 (human hepatoma) and K562 (human leukaemia), did not cross-react with SCLC sera, i.e. these cancer cells do not contain the antigens corresponding to the above-mentioned ATA.

To evaluate the clinical value of serum ATA, the sensitivity and specificity of the whole pattern of serum ATA found may be compared with those of established tumour markers. Whereas the specificities of all individual ATA is above $90 \%$, their sensitivities are below $22 \%$ with the exception of anti-p40-p42 for which the sensitivity is estimated as $59 \%$. However, in the case of the pattern, i.e. the combination of antibodies against p40-42 kD, p36, p30, p28, p26, and p14, the sensitivity and specificity are equal to $80 \%$ and $91 \%$, respectively (table 3 ). These values are comparable with the corresponding characteristics of such established tumour markers as neurone-specific enolase $(74 \%$ and $83 \%$, respectively) [15] and progastrin-releasing peptide $(47 \%$ and $95 \%)$ [16]. Thus, it may be concluded that the described method using the ATA pattern is about as powerful as the use of the established tumour markers previously mentioned.

It was of particular interest to know whether the ATA pattern was present in sera of patients with various pulmonary disorders, irradiated during the liquidation of the consequences of the Chernobyl nuclear power plant accident (a high-risk group in lung cancer). It turned out that antibodies against p40-p42 in these patients had the frequencies which held an intermediate position between sera of SCLC patients and healthy individuals. This group of patients continue to be under careful medical observation, in particular the dynamics of the ATA pattern are measured, to answer the question as to whether the pattern can predict the consequent development of lung cancer.

In connection with the results obtained in the current study, a question remains, "What is the nature of the antigens corresponding to the above-mentioned antitumour antibodies?". The present work creates a foundation for further identification of the nature of the antigens revealed and their clinical application. The use of two-dimensional electrophoresis, possibly with additional procedures of purification, in combination with the matrix-assisted laser desorption method, could help to identify these antigens.

Acknowledgements. The authors are indebted to K. Rawson-Jones (Faculty of Foreign Languages, M.V. Lomonosov Moscow State University) and E.V. Bragina for helping in the preparation of this article, and to E.E. Skorikova and I.P. Vorojeikina for their assistance. 


\section{References}

1. Posner JB, Dalmau JO. Paraneoplastic syndromes affecting the central nervous system. Annu Rev Med 1997; 48: 157-166.

2. Grunwald GB, Klein R, Simmonds MA, Kornguth SE. Autoimmune basis for visual paraneoplastic syndrome in patients with small-cell lung carcinoma. Lancet 1985; 1: 658-661.

3. Kornguth SE, Kalinke T, Grunwald GB, Schutta H, Dahl D. Anti-neurofilament antibodies in the sera of patients with small cell carcinoma of the lung and with visual paraneoplastic syndrome. Cancer Res 1986; 46: 2588-2595.

4. Thirkill CE, Roth AM, Keltner JL. Cancer-associated retinopathy. Arch Ophthalmol 1987; 105: 372-375.

5. Budde-Steffen C, Anderson NE, Rosenblum MK, Posner JB. Expression of an antigen in small cell lung carcinoma lines detected by antibodies from patients with paraneoplastic dorsal root ganglionpathy. Cancer Res 1988; 48: 430-434.

6. Tureci O, Sahin U, Pfreundschuh M. Serological analysis of human tumor antigens: molecular definition and implications. Mol Med Today 1997; 3: 342 349.

7. Hersh B, Dalmau J, Dangond E, Gultekin S, Geller E, Wen PY. Paraneoplastic opsoclonus-myoclonus associated with anti-Hu antibody. Neurology 1994; 44: 1754-1755.

8. Dalmau J, Furneaux NM, Gralla RJ, Kris MG, Posner JB. Detection of the anti-Hu antibody in the serum of patients with small cell lung cancer - a quantitative western blot analysis. Ann Neurol 1990; 27: $544-552$.

9. Drlicek M, Bianchi G, Bogliun G, et al. Antibodies of the anti-Yo and anti-Ri type in the absence of paraneoplastic neurological syndromes: a long-term survey of ovarian cancer patients. J Neurol 1997; 244: 85-89.

10. Bazhin AV, Shifrina ON, Savchenko MS, et al. Low titre autoantibodies against recoverin in sera of patients with small cell lung cancer but without a loss of vision. Lung Cancer 2001; 34: 99-104.

11. Felician O, Renard JL, Vega F, et al. Paraneoplastic cerebellar degeneration with anti-Yo antibody in a man. Neurology 1995; 45: 1226-1227.

12. Honnorat J, Trillet M, Antoine JC, et al. Paraneoplastic opsomyoclonus, cerebellar ataxia and encephalopathy associated with anti-Purkinje cell antibodies. J Neurol 1997; 244: 333-335.

13. Voltz R, Gultekin SH, Rosenfeld MR, et al. A serologic marker of paraneoplastic limbic and brainstem encephalitis in patients with testicular cancer. N Engl J Med 1999; 340: 1788-1795.

14. Gazdar AF, Mulshine JL. Biological, molecular, and clinical markers for the diagnosis and typing of lung cancer. Immunol Ser 1990; 53: 453-468.

15. Pinson P, Joos G, Watripont P, Brusslelle G, Pauwels R. Serum neuron-specific enolase as a tumor marker in the diagnosis and follow-up of small-cell lung cancer. Respiration 1997; 64: 102-107.

16. Stieber P, Dienemann H, Schalhorn A, et al. Progastrin-releasing peptide (ProGRP) - a useful marker in small cell lung carcinomas. Anticancer Res 1999; 19: 2673-2678. 